\title{
Comparative Analysis of Cycloid Bevel Gear in Different Amount of Modification
}

\author{
Jianjun Yang ${ }^{1,2, a}$, Penghui Chen ${ }^{2, b}$ \\ ${ }^{1}$ School of Mechatronics Engineering, Henan University of Science \& Technology, Luoyang \\ 471003, China \\ ${ }^{2}$ Collaborative Innovation Center of Machinery Equipment Advanced Manufacturing of Henan \\ Province, Luoyang 471003, China \\ ahohooyang@126.com, b418272398@qq.com
}

\begin{abstract}
Keywords: Modification, Cycloid Bevel Gear, Discrete Points, Finite Element Analysis
Abstract. In order to improve the meshing performance of precision forging cycloid bevel gear, the modified method of gear based on the deviation surface is provided in this paper. On the basis of generating method for pinion with tilted cutter the gear tooth surface is derived according to the fully conjugate principle. In term of the pre-determined contact path and parabolic transmission error, the target gear surfaces are achieved by superposing the two different modifications on the conjugate gear surfaces utilizing Ease-off respectively. In the end, the feasibility of the modification method proposed is verified by means of tooth contact analysis (TCA) and finite element analysis (FEA).
\end{abstract}

\section{Introduction}

Faced-hobbed cycloid bevel gears are widely applied in main drive mechanism of vehicles, it is difficult to manufacture moulds directly due to the small taper angle, however, the gear could manufacture moulds directly, precision forging is used to enhance productivity, the moulds of gear are modified to improve the meshing quality of the gear pairs.

Local synthesis method proposed by Litvin could precontrol the meshing performance of the area closed to the reference point[1]. Zongde Fang et al. put forward a initiative design method of spiral bevel gear tooth surface to achieve the control of tooth surface meshing quality in the whole process[2]. Stadtfeld modified the surface of the pinion based on the ease-off deviation topography in view of the pinion single-side machining method of the pinion[3]. Artoni,Alessio compensated the surface deviation of the spiral bevel gear based on the ease-off deviation topography and modified the surface of the pinion[4]. Shih proposed the method of tooth flank modification based on the ease-off topography on the consideration of the face-hobbing method[5,6].

Methods introduced in literatures are for pinion tooth surface modification, for this problem, this paper proposes a cycloid bevel gear tooth surface modification method, the gear surface completely conjugated with pinion surface is built, through the preset parabolic transmission error, and the preset angle of contact path and with root cone is constant, by changing the coefficients of ease-off surface to adjust the amount of modification, the variation of tooth meshing area and contact stress are compared under different modification amount through loaded finite element contact analysis, which build the foundation for improving the forging precision and contact quality of the cycloid bevel gear.

\section{Preset Transmission Error and Contact Path}

The concept of an imaginary generating gear is used to explain the generating cutting process of cycloid bevel gears, the tooth surfaces of imaginary generating gear and work gear are full conjugate, tooth surfaces contact linearly and the processed surface is solved by the way, similarly, pinion surface as the surface of the generating gear and gear surface as the processed surface when solving a gear pair, which could calculate the gear surface conjugated with the pinion surface.

According to the rotation projection principle, the position vector and normal vector of discrete point of the conjugate gear are calculated by least square method. Rotational angle of the gear and 
pinion $\varphi_{2}, \varphi_{1}$ meet the following transmission relationship in the process of meshing, as in Eq. 1 .

$$
\varphi_{2}=\frac{Z_{1}}{Z_{2}}\left(\varphi_{1}-\varphi_{1}^{0}\right)+\varphi_{2}^{0}+k\left(\varphi_{1}-\varphi_{1}^{0}\right)^{2}
$$

Where $k\left(\varphi_{1}-\varphi_{1}^{0}\right)^{2}$ is preset parabolic transmission error, $k$ is associated with the opening size of the transmission error curve, parameters $\varphi_{2}^{0}$ and $\varphi_{1}^{0}$ represent the initial rotational angles of the designed reference point of the gear and pinion, $Z_{1}$ is the number of the pinion, $Z_{2}$ is the number of the gear.

Theoretically, a conjugate gear pair has better meshing performance, but is sensitive to the installation error, load concentration easily occur to damage the gear, the conjugate gear hardly meet the demand of transmission practically. Hence, completely conjugate tooth surface is modified to realize local conjugate contact. This paper adds the certain amount of modification to the normal direction of discrete points of the fully conjugate gear surface, then the discrete point coordinates of the modified gear flank are solved to accomplish the modification of the conjugate gear.

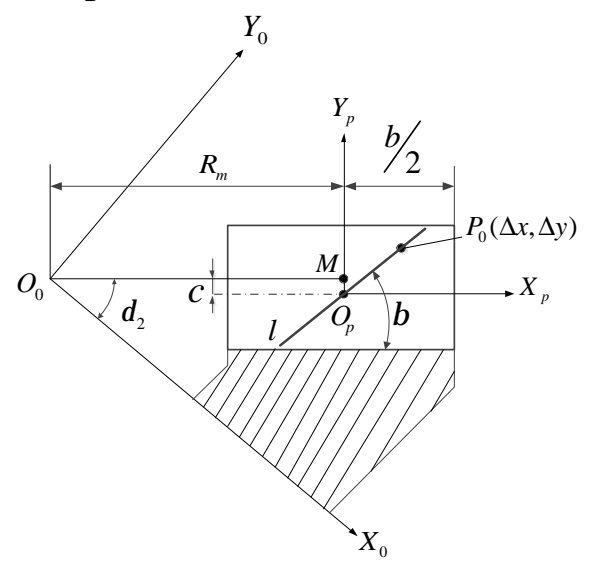

Fig. 1 The setting of the contact path

The mean point of the tooth surface is taken as coordinate system origin to establish local coordinate system $S_{p}$ along the tooth lengthwise direction that coincide with the $X$-axis, as Fig. 1 shows, point $P_{0}$ is expressed in $S_{p}$ according to the coordinate transformation, as in Eq. 2.

$$
\mathbf{r}_{p}=M_{p 0} \mathbf{r}_{0} \text {. }
$$

Where $M_{p 0}$ is a matrix transformation $S_{0}$ from to $S_{p} . R_{m}$ is the pitch cone mean distance of the gear, $\delta_{2}$ is pitch angle of the gear, $c$ is the distance of the cone line and middle point of tooth surface, $c=h_{f}-h / 2, h_{f}$ is the gear mean dedendum, $h$ is the mean whole depth.

A meshing trace $l$ is set through the middle point in the projection plane of the gear flank, $\beta$ is the angle of $l$ and root cone. For target gear tooth surface and pinion surface contacting at the point along the contact trace $l$ according to the scheduled transmission relation, the conjugate gear surface $\Sigma_{f}$ should be modified, the amount of modification of all points on the tooth face constitutes a difference surface, the difference surface touches the surface $\Sigma_{f}$ along the contact trace $l$ that supposed a quadric surface, so the difference surface is described as a parabolic cylinder along straight line $l$, as in Eq. 3.

$$
\Delta \delta=a(x \cdot \sin \beta-y \cdot \cos \beta)^{2} .
$$

Where $a$ is a coefficient related to the contact area length of ellipse, the values of $b$ and $\beta$ are listed in Table 1.

Target tooth surface of the gear is $\Sigma_{g}$, corresponding vector equation is $\mathbf{r}_{g}\left(u_{1}, \theta_{1}\right)$, the target tooth surface of the gear is obtained by the following equation, as in Eq. 4. 


$$
\mathbf{r}_{g}=\mathbf{r}_{f}+\Delta \delta \cdot \mathbf{n}_{f} .
$$

Table 1 The values of $b$ and $\beta$

\begin{tabular}{ccc}
\hline Coefficients & $b(-)$ & $\beta$ (deg) \\
\hline Case 1 & 0.0005 & 25 \\
Case 2 & 0.0001 & 25 \\
\hline
\end{tabular}

Table 2 Design data

\begin{tabular}{lccc}
\hline Items & $\begin{array}{c}\text { Pinion } \\
\text { (Left hand) }\end{array}$ & $\begin{array}{c}\text { Gear } \\
\text { (Right hand) }\end{array}$ \\
\hline Number of teeth (-) & 24 & 29 \\
Moudule (mm) & & 7.646462 & \\
Face width (mm) & 50 & \\
Shaft angle (deg) & & 90 & \\
Outer cone distance (mm) & 194.705485 & & 194.705485 \\
Mean cone distance (mm) & 169.705485 & & 32 \\
Mean spiral angle (deg) & 32 & 50.705485 \\
Pitch angle (deg) & 39.610689 & & 329313 \\
\hline
\end{tabular}

\section{Analysis of Example}

The paper explains the modification effect via a gear set of cycloid bevel gear, the parameters are listed in Table 2. On the one hand, the tooth surface modification is established on the basis of the discrete points, the modification result is verified by numerical method firstly, the precision digital gear tooth surface is obtained by NURBS surface, after carrying out meshing simulation analysis of the modified tooth surface based on space meshing theory, the contact path and transmission error curve are acquired, as Fig. 2 shows, the angle of the contact path and root cone $\beta$ '=26.56 deg, difference value is $1.56 \mathrm{deg}$ compared with preset angle and meet the design needs basically, the variation of modification amount is not impacting the orientation and position of contact path and transmission error curve after the analysis of the results.

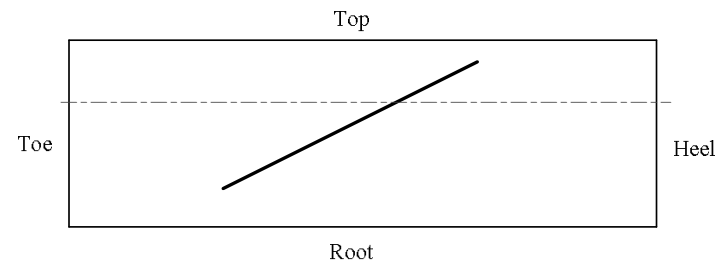

(a) Contact pattern

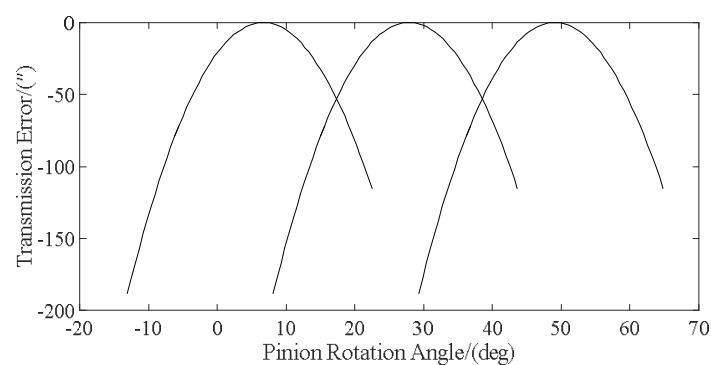

(b) Transmission error

Fig. 2 TCA results for the cycloid bevel gear set

On the other hand, three-tooth contact pairs are adopted towards conducting finite element analysis in this study, the amount of the deviation of the gear goal surface and conjugate surface in the normal direction of the conjugate gear surface is built, the certain amount of deviation is added to the normal direction of discrete points of the fully conjugate gear surface, then the discrete point coordinates of the modified gear flank are calculated, the 3D model of the modified gear is obtained after the surface reconstruction, the gear is assembled with the pinion solved by the processing parameters, the model was separated into multiple single tooth model and the finite element grid was divided. End face and side face of the tooth are respectively separated into 2D quadrilateral mesh, the unit of C3D8R is chosen for the twist less affect the accuracy of analysis and get the hexahedral mesh by means of 
sweeping.
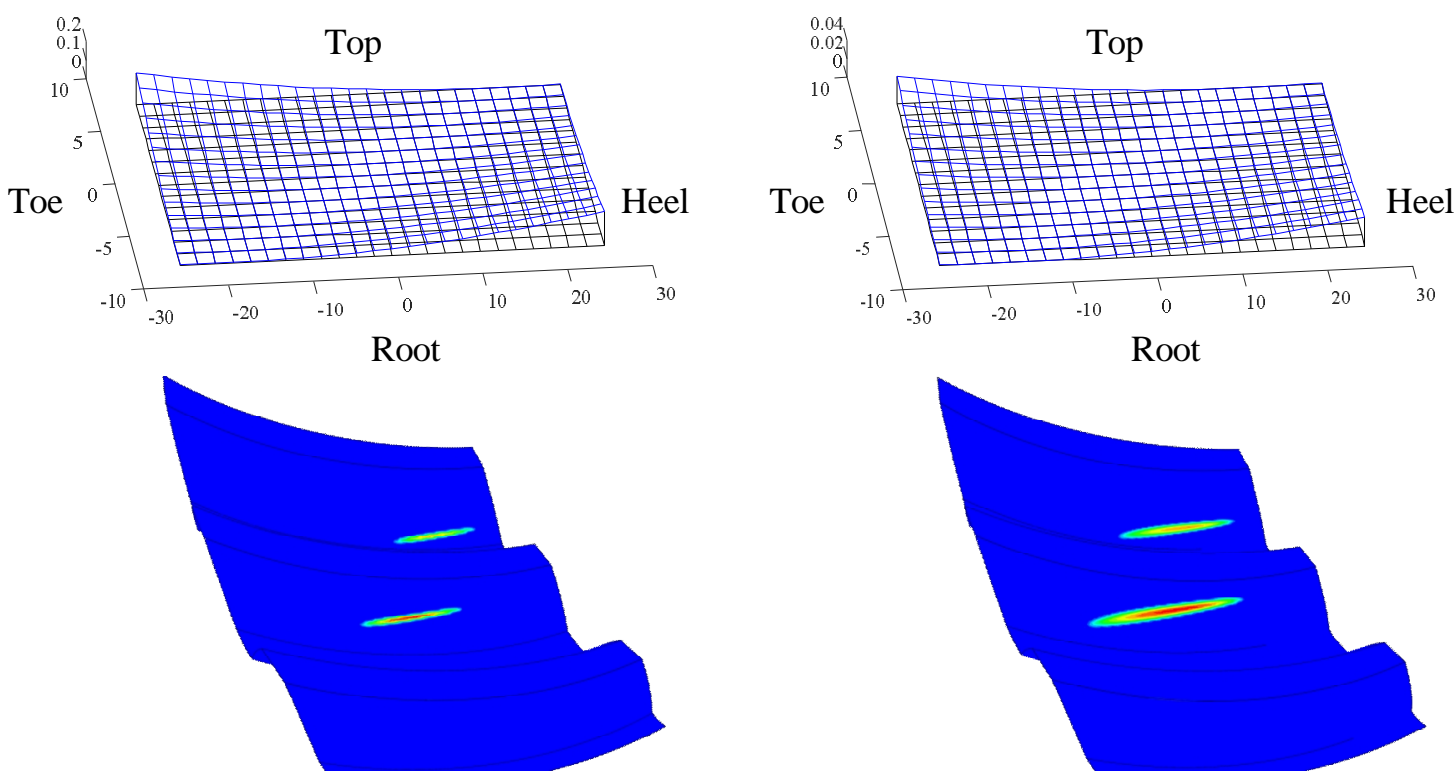

Fig. 3 Ease-off topographies and contact areas of the gear convex

In the above ease-off topographies, as Fig. 3 shows, the amount of dedendum modification is bigger than the amount of addendum modification in the heel, but opposite end is not, contact region shows bias in. On the gear convex, tooth contact path is from addendum of heel to dedendum of toe, the angle of the contact path and root cone approximately coincides with the preset value, and the size of the contact ellipse decreases as the amount of modification increasing.

\section{Conclusions}

The presented design method of tooth surface in this paper, presetting the inclined angle of contact path and parabolic transmission error on the basis of fiducial gear surface fully conjugated with the pinion, then adjusting the coefficients of ease-off surface to obtain the tooth surface of two different amount of modification, and the feasibility of the modification method proposed is verified through TCA and FEA, which lays the foundation for the research of the tooth surface meshing performance of higher-order transmission errors.

\section{Acknowledgements}

Financial assistance was provided by the National Natural Science Foundation of China (No. 51375144) and the Program for Science \& Technology Innovation Talents in Universities of Henan Province (No.15HASTIT025).

\section{References}

[1] Litvin F L, Zhang Y, Handschuh R F: Vol. 1(1991), p.1-11

[2] Xuemei Cao, Zongde Fang: Vol. 44(2008), p. 209-214

[3] Stadtfeld, H.J: Vol. 18(2001), p. 18-23

[4] Artoni A, Bracci A, Gabiccini M: Vol. 139(2009), p. 1044

[5] Shih Y P, Fong Z H: Vol. 129(2007), p. 1294-1302

[6] Shih Y P: Vol. 45(2010), p. 1108-1124 